Received: 6 June 2011, Accepted: 13 July 2011

Edited by: D. Restrepo

Reviewed by: J. H. Muñoz, Universidad del Tolima, Ibagué, Colombia; and Centro Brasileiro de Pesquisas Fisica

Licence: Creative Commons Attribution 3.0

www.papersinphysics.org

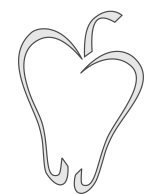

ISSN 1852-4249

\title{
Calculation of almost all energy levels of baryons
}

\begin{abstract}
Mario Everaldo de Souza ${ }^{1 *}$
It is considered that the effective interaction between any two quarks of a baryon can be approximately described by a simple harmonic potential. The problem is firstly solved in Cartesian coordinates in order to find the energy levels irrespective of their angular momenta. Then, the problem is also solved in polar cylindrical coordinates in order to take into account the angular momenta of the levels. Comparing the two solutions, a correspondence is made between the angular momenta and parities for almost all experimentally determined levels. The agreement with the experimental data is quite impressive and, in general, the discrepancy between calculated and experimental values is below $5 \%$. A couple of levels of $\Delta, N, \Sigma^{ \pm}$, and $\Omega$ present discrepacies between $6.7 \%$ and $12.5 \%[N(1655)$, $N(1440), N(1675), N(1685), N(1700), N(1710), N(1720), N(1990), N(2600), \Delta(1700)$, $\Delta(2000), \Delta(2300), \Sigma^{ \pm}(1189), \Lambda(1520), \Omega(1672)$ and $\left.\Omega(2250)\right]$.
\end{abstract}

\section{Introduction}

There are several important works that deal with the calculation of the energy levels of baryons. One of the most important ones is the pioneering work of Gasiorowicz and Rosner [1] which has calculation of baryon energy levels and magnetic moments of baryons using approximate wavefunctions. Another important work is that of Isgur and Karl [2] which strongly suggests that non-relativistic quantum mechanics can be used in the calculation of baryon spectra. Other very important attempts towards the understanding of baryon spectra are the works of Capstick and Isgur [3], Bhaduri et al. [4] Murthy et al. [5], Murthy et al. [6] and Stassat et al. [7]. Still another important work that attempts to describe baryon spectra is the recent work of Hosaka, Toki and Takayama [8] that makes use of a non-central harmonic potential (called by the authors the deformed oscillator) and is able to describe many levels. This present work describes many more levels and is more consistent in the characterization of angular momenta and parities of levels. It is an updated version of the pre-print of Ref. $[9]$.

\section{The approximation for the effec- tive potential}

The effective potential between any two quarks of a baryon is not known and thus a couple of different potentials can be found in the literature. Of course, the effective potential is the result of the attractive and repulsive forces of QCD and is completely justified because, as it is well known that the strong

\footnotetext{
*E-mail: mariodesouza.ufs@gmail.com

1 Universidade Federal de Sergipe, Departamento de Física, Av. Marechal Rondon, s/n, Campus Universitário, Jardim Rosa Elze 49100-000, São Cristovão, Brazil.
} 
Papers in Physics, vol. 3, ART. 030003 (2011) / M. E. de Souza

force becomes repulsive for very short distances, and thus repulsion and attraction can form a potential well that can be approximated with a harmonic potential about the equilibrium point. Taking into consideration the work of Isgur and Karl [2] about the use of non-relativistic quantum mechanics, and considering that the three quarks of a baryon are always on a plane, we consider that the system can be approximately described by three non-central and non-relativistic linear harmonic potentials. This is a calculation quite different from those found in the literature and explains almost all energy levels of baryons.

\section{Calculation in Cartesian coordi- nates and comparison with ex- perimental data}

The initial calculation, in which we have used Cartesian coordinates, does not, of course, consider the angular momentum of the system, that is, it does not take into account the symmetries of the system. This calculation is important for the iden- tification of the energy levels given by the experimental data, and for the assignment of the angular momenta later on. Also, it allows the prediction of many energy levels. Since each oscillator has two degrees of freedom, the energy of the system of 3 quarks is given by [10]

$$
\begin{aligned}
E_{n, m, k}= & h \nu_{1}(n+1)+h \nu_{2}(m+1) \\
& +h \nu_{3}(k+1)
\end{aligned}
$$

where $n, m, k=0,1,2,3,4, \ldots$ Of course, we identify $h \nu_{1}, h \nu_{2}, h \nu_{3}$ with the ground states of the corresponding energy levels of baryons, and thus $h \nu_{1}, h \nu_{2}, h \nu_{3}$ are equal to the masses of constituent quarks. Since we do not take isospin into account, we cannot distinguish between $N$ and $\Delta$ states, or between $\Sigma$ and $\Lambda$ states. The experimental values for the baryon levels were taken from Particle Data Group (Nakamura et al. [11]). The masses of constituent quarks are taken as $m_{u}=m_{d}=0.31 \mathrm{GeV}$, $m_{s}=0.5 \mathrm{GeV}, m_{c}=1.7 \mathrm{GeV}, m_{b}=5 \mathrm{GeV}$, and $m_{t}=174 \mathrm{GeV}$. We have, thus, the following formulas (see Table 1) for the energy levels of all known baryons up to now:

\begin{tabular}{ll}
\hline \hline Baryons & Formulas for the energy levels (in GeV) \\
\hline$N, \Delta^{-}, \Delta^{++}$ & $E_{n, m, k}=0.31(n+m+k+3)$ \\
$\Lambda^{0}, \Sigma^{+}, \Sigma^{0}, \Sigma^{-}$ & $E_{n, m, k}=0.31(n+m+2)+0.5(k+1)$ \\
$\Xi^{0}, \Xi^{-}$ & $E_{n, m, k}=0.31(n+1)+0.5(m+k+2)$ \\
$\Omega^{-}$ & $E_{n, m, k}=0.5(n+m+k+3)$ \\
$\Lambda_{c}^{+}, \Sigma_{c}^{+}, \Sigma_{c}^{++}, \Sigma_{c}^{0}$ & $E_{n, m, k}=0.31(n+m+2)+1.7(k+1)$ \\
$\Xi_{c}^{0}, \Xi_{c}^{+}$ & $E_{n, m, k}=0.31(n+1)+0.5(m+1)+1.7(k+1)$ \\
$\Omega_{c}^{0}$ & $E_{n, m, k}=0.5(n+m+2)+1.7(k+1)$ \\
$X_{c c}$ & $E_{n, m, k}=0.31(n+1)+1.7(m+k+2)$ \\
$\Lambda_{b}^{0}$ & $E_{n, m, k}=0.31(n+m+2)+5(k+1)$ \\
$\Xi_{b}^{0}, \Xi_{b}^{-}$ & $E_{n, m, k}=0.31(n+1)+0.5(m+1)+5(k+1)$ \\
$\Omega_{b}^{-}$ & $E_{n, m, k}=0.5(n+m+2)+5(k+1)$ \\
\hline \hline
\end{tabular}

Table 1: Formulas for most energy levels of all baryons.

In Tables 1 to $11, E_{C}$ is the calculated value by the above formulas, $E_{M}$ is the measured value and the error is given by Error $=100 \% \times \mid E_{M}-$ $E_{C} \mid / E_{C}$.

Within the scope of our simple calculation, many levels are degenerate, of course. Further calculations, taking into account spin-orbit and spin-spin effects, should lift part of the degeneracy. We notice that these effects are quite complex. States such as $1.70(N) D_{13}$ and $1.70(\Delta) D_{33}$ clearly show that isospin does not play an important role in the splitting of the levels. In general, the Error is below $5 \%$. 
Papers in Physics, vol. 3, ART. 030003 (2011) / M. E. de Souza

\begin{tabular}{llllll}
\hline \hline State $(n, m, k)$ & $E_{C}(\mathrm{GeV})$ & $E_{M}(\mathrm{GeV})$ & Error $(\%)$ & $L_{2 I, 2 J}$ & Parity \\
\hline $0,0,0$ & 0.93 & $0.938(N)$ & 0.9 & $P_{11}$ & + \\
\hline$n+m+k=1$ & 1.24 & $1.232(\Delta)$ & 0.6 & $P_{33}$ & + \\
\hline$n+m+k=2$ & 1.55 & $1.44(N)$ & 7.1 & $P_{11}$ & + \\
$n+m+k=2$ & 1.55 & $1.52(N)$ & 1.9 & $D_{13}$ & - \\
$n+m+k=2$ & 1.55 & $1.535(N)$ & 1.0 & $S_{11}$ & - \\
$n+m+k=2$ & 1.55 & $1.6(\Delta)$ & 3.1 & $P_{33}$ & + \\
$n+m+k=2$ & 1.55 & $1.62(\Delta)$ & 4.5 & $S_{31}$ & - \\
$n+m+k=2$ & 1.55 & $1.655(N)$ & 6.7 & $S_{11}$ & - \\
$n+m+k=2$ & 1.55 & $1.675(N)$ & 8.1 & $D_{15}$ & - \\
$n+m+k=2$ & 1.55 & $1.685(N)$ & 8.7 & $F_{15}$ & + \\
$n+m+k=2$ & 1.55 & $1.70(N)$ & 9.7 & $D_{13}$ & - \\
$n+m+k=2$ & 1.55 & $1.70(\Delta)$ & 9.7 & $D_{33}$ & - \\
$n+m+k=2$ & 1.55 & $1.72(N)$ & 11.0 & $P_{13}$ & + \\
\hline$n+m+k=3$ & 1.86 & $1.71(N)$ & 8.1 & $P_{11}$ & + \\
$n+m+k=3$ & 1.86 & $1.90(N)$ & 2.2 & $P_{13}$ & + \\
$n+m+k=3$ & 1.86 & $1.90(\Delta)$ & 2.2 & $S_{31}$ & - \\
$n+m+k=3$ & 1.86 & $1.905(\Delta)$ & 2.4 & $F_{35}$ & + \\
$n+m+k=3$ & 1.86 & $1.91(\Delta)$ & 2.7 & $P_{31}$ & + \\
$n+m+k=3$ & 1.86 & $1.92(\Delta)$ & 3.2 & $P_{33}$ & + \\
$n+m+k=3$ & 1.86 & $1.93(\Delta)$ & 3.8 & $D_{35}$ & - \\
$n+m+k=3$ & 1.86 & $1.94(\Delta)$ & 4.3 & $D_{33}$ & - \\
$n+m+k=3$ & 1.86 & $2.0(N)$ & 7.5 & $F_{15}$ & + \\
\hline$n+m+k=4$ & 2.17 & $1.95(\Delta)$ & 10.1 & $F_{37}$ & + \\
$n+m+k=4$ & 2.17 & $1.99(N)$ & 8.3 & $F_{17}$ & + \\
$n+m+k=4$ & 2.17 & $2.00(\Delta)$ & 7.8 & $F_{35}$ & + \\
$n+m+k=4$ & 2.17 & $2.08(N)$ & 4.1 & $D_{13}$ & - \\
$n+m+k=4$ & 2.17 & $2.09(N)$ & 3.7 & $S_{11}$ & - \\
$n+m+k=4$ & 2.17 & $2.10(N)$ & 3.2 & $P_{11}$ & + \\
$n+m+k=4$ & 2.17 & $2.15(\Delta)$ & 0.9 & $S_{31}$ & - \\
$n+m+k=4$ & 2.17 & $2.19(N)$ & 0.9 & $G_{17}$ & - \\
$n+m+k=4$ & 2.17 & $2.20(N)$ & 1.4 & $D_{15}$ & - \\
$n+m+k=4$ & 2.17 & $2.20(\Delta)$ & 1.4 & $G_{37}$ & - \\
$n+m+k=4$ & 2.17 & $2.22(N)$ & 2.3 & $H_{19}$ & + \\
$n+m+k=4$ & 2.17 & $2.225(N)$ & 2.5 & $G_{19}$ & - \\
$n+m+k=4$ & 2.17 & $2.3(\Delta)$ & 6.0 & $H_{39}$ & + \\
\hline$n+m+k=5$ & 2.48 & $2.35(\Delta)$ & 5.2 & $D_{35}$ & - \\
$n+m+k=5$ & 2.48 & $2.39(\Delta)$ & 3.6 & $F_{37}$ & + \\
$n+m+k=5$ & 2.48 & $2.40(\Delta)$ & 3.2 & $G_{39}$ & - \\
$n+m+k=5$ & 2.48 & $2.42(\Delta)$ & 2.4 & $H_{3,11}$ & + \\
\hline$n+m+k=6$ & 2.79 & $2.60(N)$ & 6.8 & $I_{1,11}$ & - \\
$n+m+k=6$ & 2.79 & $2.70(N)$ & 3.2 & $K_{1,13}$ & + \\
$n+m+k=6$ & 2.79 & $2.75(\Delta)$ & 1.4 & $I_{3,13}$ & - \\
\hline$n+m+k=7$ & 3.10 & $2.95(\Delta)$ & 4.8 & $K_{3,15}$ & + \\
$n+m+k=7$ & 3.10 & $3.10(N)$ & 0 & $L_{1,15}$ & - \\
\hline \hline$+m+k=8$ & 3.21 & $?$ & $?$ & $?$ & $?$ \\
\hline
\end{tabular}

Table 2: Energy levels of baryons $N$ and $\Delta$. 
Papers in Physics, vol. 3, ART. 030003 (2011) / M. E. de Souza

\begin{tabular}{llllll}
\hline \hline State $(n, m, k)$ & $E_{C}(\mathrm{GeV})$ & $E_{M}(\mathrm{GeV})$ & Error $(\%)$ & $L_{2 I, 2 J}$ & Parity \\
\hline $0,0,0$ & 1.12 & $1.116(\Lambda)$ & 0.4 & $P_{01}$ & + \\
$0,0,0$ & 1.12 & $1.189\left(\Sigma^{ \pm}\right)$ & 6.2 & $P_{11}$ & + \\
$0,0,0$ & 1.12 & $1.193\left(\Sigma^{0}\right)$ & 6.5 & $P_{11}$ & + \\
\hline$n+m=1, k=0$ & 1.43 & $1.385(\Sigma)$ & 3.2 & $P_{13}$ & + \\
$n+m=1, k=0$ & 1.43 & $1.405(\Lambda)$ & 1.7 & $S_{01}$ & - \\
$n+m=1, k=0$ & 1.43 & $1.48(\Sigma)$ & 3.5 & $?$ & $?$ \\
\hline $0,0,1$ & 1.62 & $1.52(\Lambda)$ & 6.2 & $D_{03}$ & - \\
$0,0,1$ & 1.62 & $1.56(\Sigma)$ & 3.7 & $?$ & + \\
$0,0,1$ & 1.62 & $1.58(\Sigma)$ & 2.5 & $D_{13}$ & - \\
$0,0,1$ & 1.62 & $1.60(\Lambda)$ & 1.2 & $P_{01}$ & + \\
$0,0,1$ & 1.62 & $1.62(\Sigma)$ & 0 & $S_{11}$ & - \\
$0,0,1$ & 1.62 & $1.66(\Sigma)$ & 2.5 & $P_{11}$ & + \\
$0,0,1$ & 1.62 & $1.67(\Lambda)$ & 3.1 & $S_{01}$ & - \\
\hline$n+m=2, k=0$ & 1.74 & $1.67(\Sigma)$ & 4.0 & $D_{13}$ & - \\
$n+m=2, k=0$ & 1.74 & $1.69(\Lambda)$ & 2.9 & $D_{03}$ & - \\
$n+m=2, k=0$ & 1.74 & $1.69(\Sigma)$ & 2.9 & $?$ & $?$ \\
$n+m=2, k=0$ & 1.74 & $1.75(\Sigma)$ & 0.6 & $S_{11}$ & - \\
$n+m=2, k=0$ & 1.74 & $1.77(\Sigma)$ & 1.7 & $P_{11}$ & + \\
$n+m=2, k=0$ & 1.74 & $1.775(\Sigma)$ & 2.0 & $D_{15}$ & - \\
$n+m=2, k=0$ & 1.74 & $1.80(\Lambda)$ & 3.4 & $S_{01}$ & - \\
$n+m=2, k=0$ & 1.74 & $1.81(\Lambda)$ & 4.0 & $P_{01}$ & + \\
$n+m=2, k=0$ & 1.74 & $1.82(\Lambda)$ & 4.6 & $F_{05}$ & + \\
$n+m=2, k=0$ & 1.74 & $1.83(\Lambda)$ & 5.2 & $D_{05}$ & - \\
\hline$n+m=1, k=1$ & 1.93 & $1.84(\Sigma)$ & 4.7 & $P_{13}$ & + \\
$n+m=1, k=1$ & 1.93 & $1.88(\Sigma)$ & 2.6 & $P_{11}$ & + \\
$n+m=1, k=1$ & 1.93 & $1.89(\Lambda)$ & 2.1 & $P_{03}$ & + \\
$n+m=1, k=1$ & 1.93 & $1.915(\Sigma)$ & 0.8 & $F_{15}$ & + \\
$n+m=1, k=1$ & 1.93 & $1.94(\Sigma)$ & 0.5 & $D_{13}$ & - \\
\hline$n+m=3, k=0$ & 2.05 & $2.00(\Lambda)$ & 2.5 & $?$ & $?$ \\
$n+m=3, k=0$ & 2.05 & $2.00(\Sigma)$ & 2.5 & $S_{11}$ & - \\
$n+m=3, k=0$ & 2.05 & $2.02(\Lambda)$ & 1.5 & $F_{07}$ & + \\
$n+m=3, k=0$ & 2.05 & $2.03(\Sigma)$ & 1.0 & $F_{17}$ & + \\
$n+m=3, k=0$ & 2.05 & $2.07(\Sigma)$ & 1.0 & $F_{15}$ & + \\
$n+m=3, k=0$ & 2.05 & $2.08(\Sigma)$ & 1.5 & $P_{13}$ & + \\
\hline $0,0,2$ & 2.12 & $2.10(\Sigma)$ & 0.9 & $G_{17}$ & - \\
$0,0,2$ & 2.12 & $2.10(\Lambda)$ & 0.9 & $G_{07}$ & - \\
$0,0,2$ & 2.12 & $2.11(\Lambda)$ & 0.5 & $F_{05}$ & + \\
\hline$n+m=2, k=1$ & 2.24 & $2.25(\Sigma)$ & 0.5 & $?$ & $?$ \\
\hline$n+m=4, k=0$ & 2.36 & $2.325(\Lambda)$ & 1.5 & $D_{03}$ & - \\
$n+m=4, k=0$ & 2.36 & $2.35(\Lambda)$ & 0.4 & $H_{09}$ & + \\
\hline$n+m=1, k=2$ & 2.43 & $2.455(\Sigma)$ & 1.0 & $?$ & $?$ \\
\hline$n+m=3, k=1$ & 2.55 & $2.585(\Lambda)$ & 1.4 & $?$ & $?$ \\
\hline \hline
\end{tabular}

Table 3: Energy levels of $\Sigma$ and $\Lambda$. 
Papers in Physics, vol. 3, ART. 030003 (2011) / M. E. de Souza

\begin{tabular}{llllll}
\hline \hline State $(n, m, k)$ & $E_{C}(\mathrm{GeV})$ & $E_{M}(\mathrm{GeV})$ & Error $(\%)$ & $L_{2 I, 2 J}$ & Parity \\
\hline \hline $0,0,3$ & 2.62 & $2.62(\Sigma)$ & 0 & $?$ & $?$ \\
\hline$n+m=5, k=0$ & 2.67 & $?$ & $?$ & $?$ & $?$ \\
\hline$n+m=2, k=2$ & 2.74 & $?$ & $?$ & $?$ & $?$ \\
\hline$n+m=4, k=1$ & 2.86 & $?$ & $?$ & $?$ & $?$ \\
\hline$n+m=1, k=3$ & 2.93 & $?$ & $?$ & $?$ & $?$ \\
\hline$n+m=6, k=0$ & 2.98 & $3.00(\Sigma)$ & 0.7 & $?$ & $?$ \\
\hline$n+m=3, k=2$ & 3.05 & $?$ & $?$ & $?$ & $?$ \\
\hline$n+m=0, k=4$ & 3.12 & $?$ & $?$ & $?$ & $?$ \\
\hline$n+m=5, k=1$ & 3.17 & $3.17(\Sigma)$ & 0 & $?$ & $?$ \\
\hline$n+m=2, k=3$ & 3.24 & $?$ & $?$ & $?$ & $?$ \\
\hline \hline$n+m=2, k=3$ & 3.29 & $?$ & $?$ & $?$ & $?$ \\
\hline \hline
\end{tabular}

Table 3 (Cont.): Energy levels of $\Sigma$ and $\Lambda$.

\begin{tabular}{llllll}
\hline \hline State $(n, m, k)$ & $E_{C}(\mathrm{GeV})$ & $E_{M}(\mathrm{GeV})$ & Error $(\%)$ & $L_{2 I, 2 J}$ & Parity \\
\hline $0,0,0$ & 1.31 & $1.315\left(\Xi^{0}\right)$ & 0.5 & $P_{11}$ & + \\
$0,0,0$ & 1.31 & $1.321\left(\Xi^{-}\right)$ & 0.8 & $P_{11}$ & + \\
\hline $1,0,0$ & 1.62 & 1.53 & 5.6 & $P_{13}$ & + \\
$1,0,0$ & 1.62 & 1.62 & 0 & $?$ & $?$ \\
$1,0,0$ & 1.62 & 1.69 & 4.3 & $?$ & $?$ \\
\hline$n=0, m+k=1$ & 1.81 & 1.82 & 0.6 & $D_{13}$ & - \\
\hline $2,0,0$ & 1.93 & 1.95 & 1.0 & $?$ & $?$ \\
\hline$n=1, m+k=1$ & 2.12 & 2.03 & 4.2 & $?$ & $?$ \\
$n=1, m+k=1$ & 2.12 & 2.12 & 0 & $?$ & $?$ \\
\hline$n=3, m=k=0$ & 2.24 & 2.25 & 0.5 & $?$ & $?$ \\
\hline$n=0, m+k=2$ & 2.31 & 2.37 & 2.6 & $?$ & $?$ \\
\hline$n=2, m+k=1$ & 2.43 & $?$ & $?$ & $?$ & $?$ \\
\hline$n=4, m=k=0$ & 2.55 & 2.5 & 2.0 & $?$ & $?$ \\
\hline \hline
\end{tabular}

Table 4: Energy levels of $\Xi$.

\begin{tabular}{llll}
\hline \hline State $(n, m, k)$ & $E_{C}(\mathrm{GeV})$ & $E_{M}(\mathrm{GeV})$ & Error $(\%)$ \\
\hline $0,0,0$ & 1.5 & 1.672 & 11.17 \\
\hline$n+m+k=1$ & 2.0 & 2.25 & 12.5 \\
\hline$n+m+k=2$ & 2.5 & 2.38 & 4.8 \\
$n+m+k=2$ & 2.5 & 2.47 & 1.2 \\
\hline$n+m+k=3$ & 3.0 & $?$ & $?$ \\
\hline \hline
\end{tabular}

Table 5: Energy levels of $\Omega$.

\begin{tabular}{llll}
\hline \hline State $(n, m, k)$ & $E_{C}(\mathrm{GeV})$ & $E_{M}(\mathrm{GeV})$ & Error $(\%)$ \\
\hline $0,0,0$ & 2.32 & 2.285 & 1.5 \\
\hline$n+m=1, k=0$ & 2.63 & 2.594 & 0.1 \\
$n+m=1, k=0$ & 2.63 & 2.625 & 0.2 \\
\hline$n+m=2, k=0$ & 2.94 & $?$ & $?$ \\
\hline \hline
\end{tabular}

Table 6: Energy levels of $\Lambda_{c}$. 
Papers in Physics, vol. 3, ART. 030003 (2011) / M. E. de Souza

\begin{tabular}{llll}
\hline \hline State $(n, m, k)$ & $E_{C}(\mathrm{GeV})$ & $E_{M}(\mathrm{GeV})$ & Error $(\%)$ \\
\hline $0,0,0$ & 2.51 & $2.46\left(\Xi_{c}^{+}\right)$ & 2.0 \\
$0,0,0$ & 2.51 & $2.47\left(\Xi_{c}^{0}\right)$ & 1.6 \\
\hline $1,0,0$ & 2.82 & 2.79 & 1.1 \\
$1,0,0$ & 2.82 & 2.815 & 0.2 \\
\hline $0,1,0$ & 3.01 & 2.93 & 2.7 \\
$0,1,0$ & 3.01 & 2.98 & 1.0 \\
$0,1,0$ & 3.01 & 3.055 & 1.5 \\
\hline $2,0,0$ & 3.13 & 3.08 & 1.6 \\
$2,0,0$ & 3.13 & 3.123 & 0.2 \\
\hline $1,1,0$ & 3.32 & $?$ & $?$ \\
\hline $3,0,0$ & 3.44 & $?$ & $?$ \\
\hline \hline
\end{tabular}

Table 7: Energy levels of $\Xi_{c}$.

\begin{tabular}{llll}
\hline \hline State $(n, m, k)$ & $E_{C}(\mathrm{GeV})$ & $E_{M}(\mathrm{GeV})$ & Error $(\%)$ \\
\hline $0,0,0$ & 2.7 & 2.704 & 0.2 \\
\hline$n+m=1, k=0$ & 3.2 & $?$ & $?$ \\
\hline$n+m=2, k=0$ & 3.7 & $?$ & $?$ \\
\hline \hline
\end{tabular}

Table 8: Energy levels of $\Omega_{c}$.

\begin{tabular}{llll}
\hline \hline State $(n, m, k)$ & $E_{C}(\mathrm{GeV})$ & $E_{M}(\mathrm{GeV})$ & Error $(\%)$ \\
\hline $0,0,0$ & 5.62 & 5.6202 & 0.004 \\
\hline$n+m=1, k=0$ & 5.93 & $?$ & $?$ \\
\hline$n+m=2, k=0$ & 6.24 & $?$ & $?$ \\
\hline \hline
\end{tabular}

Table 9: Energy levels of $\Lambda_{b}^{0}$.

\begin{tabular}{llll}
\hline \hline State $(n, m, k)$ & $E_{C}(\mathrm{GeV})$ & $E_{M}(\mathrm{GeV})$ & Error $(\%)$ \\
\hline $0,0,0$ & 5.81 & $5.79\left(\Xi_{b}^{0}\right)$ & 0.2 \\
$0,0,0$ & 5.81 & $5.79\left(\Xi_{b}^{-}\right)$ & 0.2 \\
\hline $1,0,0$ & 6.12 & $?$ & $?$ \\
\hline $0,1,0$ & 6.31 & $?$ & $?$ \\
\hline \hline
\end{tabular}

Table 10: Energy levels of $\Xi_{b}$.

\begin{tabular}{llll}
\hline \hline State $(n, m, k)$ & $E_{C}(\mathrm{GeV})$ & $E_{M}(\mathrm{GeV})$ & Error $(\%)$ \\
\hline $0,0,0$ & 6.0 & 6.071 & 1.2 \\
\hline$n+m=1, k=0$ & 6.5 & $?$ & $?$ \\
\hline$n+m=2, k=0$ & 7.0 & $?$ & $?$ \\
\hline \hline
\end{tabular}

Table 11: Energy levels of $\Omega_{b}$. 
Papers in Physics, vol. 3, ART. 030003 (2011) / M. E. de Souza

We can predict the energy levels of many heavy baryons, probably already found by the LHC or to be found in the near future. There are, for example, the baryon levels (in $\mathrm{GeV}$ ):

- $s c c, E_{n, m, k}=0.5(n+1)+1.7(m+k+2)$;

- $c c c, E_{n, m, k}=1.7(n+m+k+3)$;

- $c c b, E_{n, m, k}=1.7(n+m+2)+5(k+1)$;

- $c b b, E_{n, m, k}=1.7(n+1)+5(m+k+2)$;

- etc.

\section{Calculation in polar cylindri- cal coordinates and comparison with experimental data}

In order to take into account angular momentum and parity, we have to use spherical or polar coordinates. Since the 3 quarks of a baryon are always in a plane, we can use polar coordinates and choose the $\mathrm{Z}$ axis perpendicular to this plane. Now the eigenfunctions are eigenfunctions of the orbital angular momentum. Thus, we have three oscillators in a plane and we consider them to be independent. Using again the non-relativistic approximation, the radial Schrödinger equation for the stationary states of each oscillator is given by $[12,13]$

$$
\begin{aligned}
& {\left[-\frac{\hbar^{2}}{2 \mu}\left(\frac{\partial^{2}}{\partial \rho^{2}}+\frac{1}{\rho} \frac{\partial}{\partial \rho}-\frac{m_{z}}{\rho^{2}}\right)\right.} \\
& \left.\quad+\frac{1}{2} \mu \omega^{2} \rho^{2}\right] R_{E m}(\rho)=E R_{E m}(\rho)
\end{aligned}
$$

where $m_{z}$ is the quantum number associated with $L_{z}, \mu$ is the reduced mass of the oscillator, and $\omega$ is the oscillator frequency. Therefore, we have three independent oscillators with orbital angular momenta $\vec{L}_{1}, \vec{L}_{2}$ and $\vec{L}_{3}$ whose $Z$ components are $L_{z 1}, L_{z 2}$ and $L_{z 3}$. Of course, the system has total orbital angular momentum $\vec{L}=\vec{L}_{1}+\vec{L}_{2}+\vec{L}_{3}$ and each $\vec{L}_{i}$ has a quantum number $l_{i}$ associated with it. The eigenvalues of the energy levels are given by $[12,13]$

$$
\begin{aligned}
E= & \left(2 r_{1}+\left|m_{z 1}\right|+1\right) h \nu_{1}+\left(2 r_{2}+\left|m_{z 2}\right|+1\right) h \nu_{2} \\
& +\left(2 r_{3}+\left|m_{z 3}\right|+1\right) h \nu_{3}
\end{aligned}
$$

in which $r_{i}=0,1,2, \ldots$ and it is a radial quantum number, and $\left|m_{z i}\right|=0,1, \ldots, l_{i}$. Comparing equation (3) with equation (1), we have $n=2 r_{1}+\left|m_{z 1}\right|$; $m=2 r_{2}+\left|m_{z 2}\right| ; k=2 r_{3}+\left|m_{z 3}\right|$. Let us recall that if we have three angular momenta $\vec{L}_{1}, \vec{L}_{2}$ and $\vec{L}_{3}$ associated to the quantum numbers $l_{1}, l_{2}$ and $l_{3}$, the total orbital angular momentum $\vec{L}$ is described by the quantum number $L$ given by

$$
l_{1}+l_{2}+l_{3} \geq L \geq|| l_{1}-l_{2}\left|-l_{3}\right|
$$

where $l_{1} \geq\left|m_{z 1}\right| ; l_{2} \geq\left|m_{z 2}\right| ; l_{3} \geq\left|m_{z 3}\right|$.

Because the three quarks are on a plane, only $r_{i}$ and $m_{z i}$ are good quantum numbers, that is, $l_{i}$ are not good quantum numbers and their possible values are found indirectly by means of $m_{z i}$ due to the condition $l_{i} \geq m_{z i}$. This means that the upper values of $l_{i}$ cannot be found from the model, and as a consequence, the upper value of $L$ cannot be found either. We only determine the values of $L$ comparing the experimental results of the energies of the baryon states with the energy values calculated by $E_{n m k}$. This is a limitation of the model. The other models have many limitations too. For example, in the Deformed Oscillator Model some quantum numbers are not good either and are only approximate and there is not a direct relation between $N$ and $L$ where $N$ is the total quantum number. In a certain way, a baryon is a tri-atomic molecule of three quarks and thus some features of molecules may show up and that is indeed the case.

Taking into account spin, we form the total angular momentum $\vec{J}=\vec{L}+\vec{S}$ whose quantum numbers are $J=L \pm s$ where $s=1 / 2,3 / 2$. As we will see, we will be able to describe almost all baryon levels.

As in the case of the rotational spectra of triatomic molecules [14], due to the couplings of the different angular momenta, it is expected that there should exist a minimum value of $J=K$ for the total angular momentum and, thus, $J$ should have the possible values $J=K, K+1, K+2, K+3, \ldots$. But in the case of baryons, this feature does not always appear to happen.

\section{i. Baryons $N$ and $\Lambda$}

We will classify the levels according to Table 1 and take $J=L \pm 1 / 2$ or $J=L \pm 3 / 2(\Delta)$. 
a. Level $(n=m=k=0 ; 0.93 \mathrm{GeV})$

The first state of $N$ is the state $(n=m=k=0)$ with energy $0.93 \mathrm{GeV}$. Therefore, in this case $l_{1}=$ $l_{2}=l_{3}=0$ and thus $L=0$. This is the positive parity state $P_{11}$

\begin{tabular}{llll}
\hline \hline$L$ & $N$ & $\Delta$ & Parity \\
\hline 0 & $0.938 P_{11}$ & & + \\
\hline \hline
\end{tabular}

\section{b. Level $(n=m=k=1 ; 1.24 \mathrm{GeV})$}

This is the first state of $\Lambda$. As $n+m+k=1$, we have $2 r_{1}+\left|m_{z 1}\right|+2 r_{2}+\left|m_{z 2}\right|+2 r_{3}+\left|m_{z 3}\right|=1$, and thus $\left|m_{z 1}\right|+\left|m_{z 2}\right|+\left|m_{z 3}\right|=1$, and $l_{1}+l_{2}+l_{3} \geq 1$, and we can choose the sets $\left|m_{z 1}\right|=1,\left|m_{z 2}\right|=$ $\left|m_{z 3}\right|=0 ;\left|m_{z 2}\right|=1,\left|m_{z 1}\right|=\left|m_{z 3}\right|=0 ;\left|m_{z 3}\right|=1$, $\left|m_{z 1}\right|=\left|m_{z 2}\right|=0$, and $l_{1}=1, l_{2}=l_{3}=0$ or $l_{2}=1, l_{1}=l_{3}=0$ or still $l_{3}=1, l_{1}=l_{2}=0$ which produce $L \geq 0$ (ground state) and thus the level

\begin{tabular}{llll}
\hline \hline$L$ & $N$ & $\Delta$ & Parity \\
\hline 0 & & $1.232 P_{33}$ & + \\
\hline \hline
\end{tabular}

c. Level $(n=m=k=2 ; 1.55$ GeV)

In this case $n=m=k=2=2 r_{1}+\left|m_{z 1}\right|+2 r_{2}+$ $\left|m_{z 2}\right|+2 r_{3}+\left|m_{z 3}\right|$. This means that $\left|m_{z 1}\right|+\left|m_{z 2}\right|+$ $\left|m_{z 3}\right|=2,0$ and we have the sets of possible values of $l_{1}, l_{2}, l_{3}$

\begin{tabular}{ccccc}
\hline \hline$l_{1}, l_{2}, l_{3}$ & $2,0,0$ & $0,2,0$ & $0,0,2$ & $1,1,0$ \\
\hline$L$ & 2 & 2 & 2 & $0,1,2$ \\
\hline \hline$l_{1}, l_{2}, l_{3}$ & $1,0,1$ & $0,1,1$ & $0,0,0$ & \\
\hline$L$ & $0,1,2$ & $0,1,2$ & 0 & \\
\hline \hline
\end{tabular}

in which the second row presents the values of $L$ that satisfy the condition $l_{1}+l_{2}+l_{3} \geq 2,0$. As 2 is a lower bound, we can also have $L=3$. There are, therefore, the following possible states

\begin{tabular}{llll}
\hline \hline$L$ & $N$ & $\Delta$ & Parity \\
\hline 0 & $1.44 P_{11}$ & $1.6 P_{33}$ & + \\
\hline 1 & $1.535 S_{11} ; 1.655 S_{11}$ & $1.62 S_{31}$ & - \\
& $1.52 D_{13} ; 1.7 D_{13}$ & & - \\
\hline 2 & $1.72 P_{13}$ & $?$ & + \\
& $1.685 F_{15}$ & & \\
\hline 3 & $1.675 D_{15}$ & $1.70 D_{33}$ & - \\
\hline \hline
\end{tabular}

d. Level $(n=m=k=3 ; 1.86$ GeV $)$

Since $n=m=k=3=2 r_{1}+\left|m_{z 1}\right|+2 r_{2}+\left|m_{z 2}\right|+$ $2 r_{3}+\left|m_{z 3}\right|,\left|m_{z 1}\right|+\left|m_{z 2}\right|+\left|m_{z 3}\right|=3,1$, and thus $l_{1}+l_{2}+l_{3} \geq 3,1$. We have, therefore, the possibilities $L=4,3,2,1,0$ because of the condition $L \geq|| l_{1}-l_{2}\left|-l_{3}\right|$ and we can arrange the levels in the form

\begin{tabular}{llll}
\hline \hline$L$ & $N$ & $\Delta$ & Parity \\
\hline 0 & $1.71 P_{11}$ & $1.91 P_{31}$ & + \\
\hline 1 & & $1.90 S_{31}$ & - \\
& & $1.93 D_{35}$ & - \\
\hline 2 & $1.90 P_{13}$ & $?$ & + \\
& $2.0 F_{15}$ & & + \\
\hline 3 & & $1.92 P_{33}, 1.94 D_{33}$ & - \\
\hline 4 & & $1.905 F_{35}$ & + \\
\hline \hline
\end{tabular}

e. Level $(n=m=k=4 ; 2.17$ GeV)

This energy level is split in many close levels. Following what we have done above $n=m=k=$ $4=2 r_{1}+\left|m_{z 1}\right|+2 r_{2}+\left|m_{z 2}\right|+2 r_{3}+\left|m_{z 3}\right|$, which yields $\left|m_{z 1}\right|+\left|m_{z 2}\right|+\left|m_{z 3}\right|=4,2,0$, and thus $l_{1}+l_{2}+l_{3} \geq 4,2,0$. We have therefore for $L$ the possible values $L=6,5,4,3,2,1,0$ and the following assignments

\begin{tabular}{llll}
\hline \hline$L$ & $N$ & $\Delta$ & Parity \\
\hline 0 & $2.10 P_{11}$ & $?$ & + \\
\hline 1 & $2.09 S_{11}$ & $2.15 S_{31}$ & - \\
& $2.08 D_{13}$ & & \\
\hline 2 & & $1.95 F_{37}$ & + \\
\hline 3 & $2.20 D_{15}$ & $?$ & - \\
& $2.19 G_{17}$ & & - \\
\hline 4 & $2.22 H_{19}$ & $2.00 F_{35}$ & + \\
\hline 5 & $2.225 G_{19}$ & $2.20 G_{37}$ & - \\
\hline 6 & & $2.30 H_{39}$ & + \\
\hline \hline
\end{tabular}

f. Level $(n=m=k=5 ; 2.48 \mathrm{GeV})$

Doing as above $n=m=k=5=2 r_{1}+\left|m_{z 1}\right|+2 r_{2}+$ $\left|m_{z 2}\right|+2 r_{3}+\left|m_{z 3}\right|$, and thus $\left|m_{z 1}\right|+\left|m_{z 2}\right|+\left|m_{z 3}\right|=$ $5,3,1$. That is, $l_{1}+l_{2}+l_{3} \geq 5,3,1$, and so we may have $L=5,4,3,2,1,0$ because of the conditions $L \geq|| l_{1}-l_{2}\left|-l_{3}\right|$ and $l_{i} \geq\left|m_{z i}\right|$. Experimentally, though, we note that $K=1$, and hence we have the possible arrangement of levels 
Papers in Physics, vol. 3, ART. 030003 (2011) / M. E. de Souza

\begin{tabular}{|c|c|c|}
\hline $\begin{array}{ll}L & N\end{array}$ & $\Delta$ & Parity \\
\hline 1 & $2.35 D_{35}$ & - \\
\hline 2 & $2.39 F_{37}$ & + \\
\hline 3 & $2.40 G_{39}$ & - \\
\hline 4 & $2.42 H_{3,11}$ & + \\
\hline 5 & $?$ & - \\
\hline
\end{tabular}

b. Level $(n=m=k=1 ; 1.43 \mathrm{GeV})$

From $n=m=1, k=0$ we obtain $2 r_{1}+\left|m_{z 1}\right|+$ $2 r_{2}+\left|m_{z 2}\right|=1$ and $2 r_{3}+\left|m_{z 3}\right|=0$ which make $\left|m_{z 1}\right|+\left|m_{z 2}\right|=1$ and $\left|m_{z 3}\right|=0$. That is, we have the condition $l_{1}+l_{2} \geq 1, l_{3} \geq 0$ which allows us to have the possibilities $L=0,1,2$ and the states

\begin{tabular}{llll}
\hline \hline$L$ & $\Sigma$ & $\Lambda$ & Parity \\
\hline 0 & & & + \\
\hline 1 & $?$ & $1.405 S_{01}$ & - \\
\hline 2 & $1.358 P_{13}$ & $?$ & + \\
\hline \hline
\end{tabular}

$\left|m_{z 2}\right|+2 r_{3}+\left|m_{z 3}\right|$ and so $\left|m_{z 1}\right|+\left|m_{z 2}\right|+\left|m_{z 3}\right|=$ $6,4,2,0$ and thus $l_{1}+l_{2}+l_{3} \geq 6,4,2,0$, and $L$ can be $L=6,5,4,3,2,1,0$, but, from the experimental values, we note that $K=5$ and so there are the possible states

\begin{tabular}{llll}
\hline \hline$L$ & $N$ & $\Delta$ & Parity \\
\hline 5 & $2.60 I_{1,11}$ & $2.75 I_{3,13}$ & - \\
\hline 6 & $2.70 K_{1,13}$ & $?$ & + \\
\hline \hline
\end{tabular}

\section{h. Level $(n=m=k=7 ; 3.10 \mathrm{GeV})$}

From $n=m=k=7=2 r_{1}+\left|m_{z 1}\right|+2 r_{2}+$ $\left|m_{z 2}\right|+2 r_{3}+\left|m_{z 3}\right|$ we obtain $\left|m_{z 1}\right|+\left|m_{z 2}\right|+\left|m_{z 3}\right|=$ $7,5,3,1$ and hence $l_{1}+l_{2}+l_{3} \geq 7,5,3,1$, and thus the possible values for $L$ are $L=7,6,5,4,3,2,1,0$, but we note that $K=7$. Therefore, we have the list of states

\begin{tabular}{llll}
\hline \hline$L$ & $N$ & $\Delta$ & Parity \\
\hline 6 & & $2.95 K_{3,15}$ & + \\
\hline 7 & $3.10 L_{1,15}$ & $?$ & - \\
\hline \hline
\end{tabular}

\section{ii. Baryons $\Sigma$ and $\Lambda$}

We will classify the levels according to Table 2 . Again $J=L \pm 1 / 2$.

a. Level $(n=m=k=0 ; 1.12 \mathrm{GeV})$

In this state $l_{1}=l_{2}=l_{3}=0$ and thus $L=0$ and we have the state

\begin{tabular}{llll}
\hline \hline$L$ & $\Sigma$ & $\Lambda$ & Parity \\
\hline 0 & $1.189\left(\Sigma^{ \pm}\right) P_{11} ;$ & $1.116 P_{01}$ & + \\
& $1.193\left(\Sigma^{0}\right) P_{11}$ & & \\
\hline \hline
\end{tabular}

Thus, the most probable values of $L$ for the state $1.48(\Sigma)$ are $L=0,1$. Maybe $K=1$ in this case and thus $L=0$ may be suppressed.

\section{c. Level $(0,0,1 ; 1.62 \mathrm{GeV})$}

For $n=m=0$ and $k=1$ we have $\left|m_{z 1}\right|=$ $\left|m_{z 2}\right|=0$ and $\left|m_{z 3}\right|=1$. That is, we have the condition $l_{1} \geq 0, l_{2} \geq 0, l_{3} \geq 1$ which allows us to choose $l_{1}=l_{2}=0, l_{3}=1 ; l_{1}=l_{3}=1, l_{2}=0$; $l_{1}=0, l_{2}=l_{3}=1$, and thus $L \geq 0,1,2$, and the states

\begin{tabular}{llll}
\hline \hline$L$ & $\Sigma$ & $\Lambda$ & Parity \\
\hline 0 & $1.66 P_{11}$ & $1.60 P_{01}$ & + \\
\hline 1 & $1.62 S_{11}$ & $1.67 S_{01}$ & - \\
& $1.58 D_{13}$ & $1.52 D_{03}$ & - \\
\hline 2 & $?$ & $?$ & + \\
\hline \hline
\end{tabular}

d. $\quad$ Level $(n+m=2, k=0 ; 1.74 \mathrm{GeV})$

In this case $n+m=2=2 r_{1}+\left|m_{z 1}\right|+2 r_{2}+\left|m_{z 2}\right|$ and $k=2 r_{3}+\left|m_{z 3}\right|=0$, and thus we obtain $\left|m_{z 1}\right|+\left|m_{z 2}\right|=2,0$ and $\left|m_{z 3}\right|=0$. Thus, we have the conditions $l_{1}+l_{2} \geq 2,0$ and $l_{3} \geq 0$. We can then choose $l_{1}=l_{2}=l_{3}=0 ; l_{1}=l_{2}=1, l_{3}=0$; $l_{1}=2, l_{2}=l_{3}=0 ; l_{2}=2, l_{1}=l_{3}=0 ; l_{1}=3, l_{2}=$ $l_{3}=0$, and we may have thus $L=0,1,2,3$ and the assignments

\begin{tabular}{llll}
\hline \hline$L$ & $\Sigma$ & $\Lambda$ & Parity \\
\hline 0 & $1.77 P_{11}$ & $1.81 P_{01}$ & + \\
\hline 1 & $1.75 S_{11}$ & $1.80 S_{01}$ & - \\
& $1.67 D_{13}$ & $1.69 D_{03}$ & - \\
\hline 2 & $?$ & $1.82 F_{05}$ & + \\
\hline 3 & $1.775 D_{15}$ & $1.83 D_{05}$ & - \\
\hline \hline
\end{tabular}

We can then say that the state $1.69(\Sigma)$ is probably a $F_{15}$ state. 
e. $\quad$ Level $(n+m=1, k=1 ; 1.93 \mathrm{GeV})$

We have $n+m=1=2 r_{1}+\left|m_{z 1}\right|+2 r_{2}+\left|m_{z 2}\right|$ and $k=2 r_{3}+\left|m_{z 3}\right|=1$, from which we obtain $\left|m_{z 1}\right|+\left|m_{z 2}\right|=1$ and $\left|m_{z 3}\right|=1$. Hence, we have the condition $l_{1}+l_{2} \geq 1$ and $l_{3} \geq 1$. We can then have the sets $l_{1}=1, l_{2}=0, l_{3}=1$; $l_{1}=0, l_{2}=1, l_{3}=1$. Both yield $L \geq 2,1,0$ and thus we identify the states

\begin{tabular}{llll}
\hline \hline$L$ & $\Sigma$ & $\Lambda$ & Parity \\
\hline 0 & $1.88 P_{11}$ & $?$ & + \\
\hline 1 & $1.94 D_{13}$ & $?$ & - \\
\hline 2 & $1.84 P_{13}$ & $1.89 P_{03}$ & + \\
& $1.915 F_{15}$ & & + \\
\hline \hline
\end{tabular}

\section{f. Level $(n+m=3, k=0 ; 2.05 \mathrm{GeV})$}

With $n+m=3=2 r_{1}+\left|m_{z 1}\right|+2 r_{2}+\left|m_{z 2}\right|$ and $k=2 r_{3}+\left|m_{z 3}\right|=0$ we obtain $\left|m_{z 1}\right|+\left|m_{z 2}\right|=3,1$ and $\left|m_{z 3}\right|=0$, and thus the conditions $l_{1}+l_{2} \geq 3,1$ and $l_{3} \geq 0$ which yield $L \geq 4,3,2,1,0$, and the possible identification taking into account that maybe $K=1$

\begin{tabular}{llll}
\hline \hline$L$ & $\Sigma$ & $\Lambda$ & Parity \\
\hline 1 & $2.00 S_{11}$ & $?$ & - \\
\hline 2 & $2.08 P_{13}$ & $?$ & + \\
& $2.07 F_{15}$ & & \\
\hline 3 & $?$ & $?$ & - \\
\hline 4 & $2.03 F_{17}$ & $2.02 F_{07}$ & + \\
\hline \hline
\end{tabular}

g. Level $(0,0,2 ; 2.12 \mathrm{GeV})$

In this case $n=0=2 r_{1}+\left|m_{z 1}\right|, m=0=$ $2 r_{2}+\left|m_{z 2}\right|$ and $k=2=2 r_{3}+\left|m_{z 3}\right|$ and thus $\left|m_{z 1}\right|=\left|m_{z 2}\right|=0$ and $\left|m_{z 3}\right|=2,0$. Hence, we have the condition $l_{1} \geq 0, l_{2} \geq 0$ and $l_{3} \geq 2,0$. We can then choose the sets $l_{1}=l_{2}=l_{3}=0$; $l_{1}=0, l_{2}=0, l_{3}=2 ; l_{1}=0, l_{2}=0, l_{3}=3 ;$ $l_{1}=l_{2}=l_{3}=1 ; l_{1}=l_{2}=1, l_{3}=0$ which make $L \geq 3,2,1,0$, and probably $K=2$. Hence, we have the possible states

\begin{tabular}{llll}
\hline \hline$L$ & $\Sigma$ & $\Lambda$ & Parity \\
\hline 2 & $?$ & $2.11 F_{05}$ & + \\
\hline 3 & $2.10 G_{17}$ & $2.10 G_{07}$ & - \\
\hline \hline
\end{tabular}

\section{h. $\quad$ Level $(n+m=4, k=0 ; 2.36 \mathrm{GeV})$}

From $n+m=4=2 r_{1}+\left|m_{z 1}\right|+2 r_{2}+\left|m_{z 2}\right|$ and $k=$ $2 r_{3}+\left|m_{z 3}\right|=0$ we obtain $\left|m_{z 1}\right|+\left|m_{z 2}\right|=4,2,0$ and $\left|m_{z 3}\right|=0$, and thus the conditions $l_{1}+l_{2} \geq 4,2,0$ and $l_{3} \geq 0$ which produce $L \geq 4,3,2,1,0$, and the possible identification

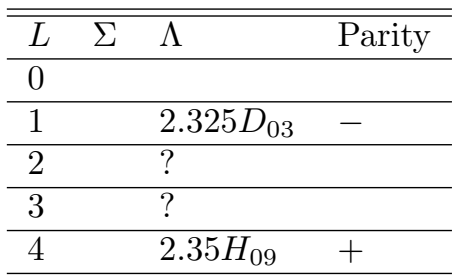

Probably in this case $K=1$ and the levels with $L=2,3$ are missing just because of a lack of experimental data. It appears that there is no state of $\Sigma$.

\section{Discussion and conclusion}

One can immediately ask about the spin degrees of freedom of the three quarks since the spin-spin interaction makes a contribution to the mass. We can say that we took care of part of it because the formulas of the energy levels depend on the three parameters $h \nu_{1}, h \nu_{2}$ and $h \nu_{3}$ which are assigned according to the masses of the constituent quarks which have already taken into account the spinspin interaction because the masses of constituent quarks are in perfect agreement with the ground state levels of baryons. Of course, the spin-spin interaction contribution depends on the energy level as is well known from the bottomonium spectrum, for example. But, as it is seen in the spectrum of bottomonium, the spin-spin contribution diminishes as the energy of the level increases. In bottomonium, the difference between the energies of $\eta_{b}(1 S)$ and $\Upsilon(1 S)$ is about $69.4 \mathrm{MeV}$, while between $\eta_{b}(2 S)$ and $\Upsilon(2 S)$ it is about $36.3 \mathrm{MeV}$, and between $\eta_{b}(3 S)$ and $\Upsilon(3 S)$ it is about $25.2 \mathrm{MeV}$, where we have used, for the energies of $\eta_{b}(2 S)$ and $\eta_{b}(3 S)$, the predicted values from reference [15], 9987.0 MeV and $10330 \mathrm{MeV}$, respectively. In the case of baryons, the spin-spin interaction varies from $15 \mathrm{MeV}$ to $30 \mathrm{MeV}$ for levels of $N, \Sigma, \Xi$ and $\Lambda$ [16]. Therefore, we observe that the spin-spin interaction is of the order of magnitude of the splitting beween neighboring levels. For example, the measured energy of the $D_{13}$ level of $N$ is $1.52 \mathrm{MeV}$, while our calculated value is $1.55 \mathrm{MeV}$, and thus the difference is $0.03 \mathrm{GeV}=30 \mathrm{MeV}$ which is of the order of the spin-spin interaction. And that is why 
Papers in Physics, vol. 3, ART. 030003 (2011) / M. E. de Souza

there are large discrepancies in the calculation of the lowest levels of $\Omega$ because in this case all quark spins are parallel and thus, the total spin-spin contribution is larger than in other baryons in which two spins are up and the other spin is down. For the lowest state of $\Omega$, the discrepancy is about 1.672 $\mathrm{GeV}-1.5 \mathrm{GeV}=0.172 \mathrm{GeV}=172 \mathrm{MeV}$. This is actually the worse calculation. But we either consider the mass of constituent quarks or we try to find tentative values for the masses of quarks like is done in QCD models which use a quite different range of arbitrary quark masses. The use of the constituent quark mass is completely justifiable in our case because we do not attempt to calculate at all the splitting between neighboring baryon levels. Such calculation can be made in the future upon improving the present model.

We only addressed the angular momenta of $N, \Delta, \Sigma$ and $\Lambda$ due to a lack of experimental data for the other baryons. Of course, the state $n=m=$ $l=0$ is missing for the $\Delta$ particle because this corresponds to the ground state of the nucleon.

We notice that the simple model above describes almost all energy levels of baryons. The splitting for a certain $L$ is quite complex. Sometimes, there is almost no dependence on spin, such as, for example, the states of $\Sigma$ with $L=2,2.08 P_{13}$ and $2.07 F_{15}$. On the other hand, the states of $\Sigma$ with the same $L=2,1.84 P_{13}$ and $1.915 F_{15}$, present a strong spin-orbit dependence. It can just be a matter of obtaining more accurate experimental results.

It is important to observe that part of the splitting is primarily caused by the spin-orbit interaction and is very complex because, in some cases, it appears to be the normal spin-orbit and, in other cases, it appears to be the inverted (negative) spin-orbit. In the simple model above, the oscillators were considered approximately independent but there may exist some coupling among them and this can contribute to the splitting of levels. As we have discussed in the first paragraph, part of the splitting should be attributed to the spinspin interaction which was not taken into account in a detailed way. Of course, part of it was considered inside the values of the three parameters $h \nu_{1}$, $h \nu_{2}$ and $h \nu_{3}$ which are taken as the masses of the three constituent quarks of a given baryon. It is important to observe that the discrepancy between calculated and measured values diminishes as the energy increases. This fact shows that the splitting is mainly caused by the spin-spin interaction.

Another important conclusion is that with the simple model above we cannot calculate the values of $K$, and from the above results we note that it is a quite difficult task because there appears to exist no pattern with respect to this. As in the case of triatomic molecules, the values of $K$ are found from the experimental data.

As we notice, in the above tables the increase in the energy of levels allows the existence of higher values of $L$ (and $J$ ). This is an old fact and is so because equation (3) has a linear dependence on $\left|l_{z i}\right|$.

For experimentalists, the classifications above are very important and can help them in the prediction of energies and angular momenta of levels. An old version of this work that appeared in Ref. [9] predicted the energies of all levels which have lately been reported, and this is a very important fact. For example, for $\Xi_{c}$ it predicted the levels (on page 8 of [9]) with energies 2.82, 3.01 and 3.13, and since 2002 the following corresponding levels of $\Xi_{c}$ have been found: $2.815,(2.93 ; 2.98 ; 3.055)$; (3.08; $3.123)$.

As it is well known, the first order correction term of anharmonicity in an oscillator for each degree of freedom is of the form

$$
\Delta E=A\left(p+\frac{1}{2}\right)^{2}
$$

where $A$ is a constant and $p$ is a non-negative integer $(p=0,1,2,3, \ldots)$. Therefore, the calculated energies of levels with high quantum numbers would be away from the experimental values. This is not observed above and, thus, the anharmonicity should be quite low. For example, for $n+m+k=7$ of $N$ we obtain that the experimental and calculated values are the same $(3.10 \mathrm{GeV})$. In the case of $\Sigma$, we have the same kind of behavior because for $(n+m=5, k=1)$ we also have the same calculated and experimental value for $\Sigma(3.17 \mathrm{GeV})$.

The assignments of the angular momenta for some few levels are only reasonable attempts. It is the case, for example, of the level $2.0 F_{15}$ of $N$ which can belong to either the $(n+m+k=3)$ or to the $(n+m+k=4)$ levels. We chose the former because 2.0 is closer to 1.86 than to 2.17 . For the level $1.99 F_{17}$ we chose the $(n+m+k=4)$ level because it appears that the highest value of $J$ 
Papers in Physics, vol. 3, ART. 030003 (2011) / M. E. de Souza

for the level $(n+m+k=3)$ is 5 . It is a strange feature that the level $(n+m+k=5)$ only contains $\Delta^{\prime} \mathrm{s}$. Having in mind what has been justified above, we chose the $2.35 D_{35}$ level of $\Delta$ belonging to $(n+m+k=5)$ as 2.35 is closer to 2.48 than to 2.17 . The level $2.60 I_{1,11}$ of $N$ was assigned as belonging to $(n+m+k=6)$ because its energy is between $2.55 \mathrm{GeV}$ and $2.75 \mathrm{GeV}$. The level $1.74 D_{13}$ of $\Sigma$ was chosen as belonging to $(n+m=2, k=0)$ because $(0,0,1)$ already has a $D_{13}$ level for $\Sigma(1.58)$. We made similar considerations in the choice of the levels $1.83(\Lambda) D_{05}, 1.84(\Sigma) P_{13}$ and $1.94(\Sigma) D_{13}$. These ambiguities will be settled either with data with smaller widths or with a more improved model.

Some levels are not described by the simple approximation above. It is the case, for instance, of $\Xi(1530) P_{13}$ which is probably a composite of $\Xi(0,0,0) \equiv \Xi(1.31)$ with a pion excitation (that is, it is a hadronic molecule). Its decay is actually $\Xi(1.31) \pi$. In the same way, the state $\Sigma_{c}(2455)$ appears to be a composite state of $\Lambda_{c}^{+}(2285)$ and a pion excitation. The same appears to hold for the other known states of $\Lambda_{c}$.

As a whole, the model describes quite well the baryonic spectra but it is far from describing the detailed splitting which appears to be quite complex and may depend on the spin-spin interaction. It does not provide a way of calculating the values of $K$. With the acquisition of more data from other baryons, we may be able to find more patterns and to improve the model. Due to the complexity of the problem, we will probably have to go back and forth several times in the improvements of the model as it has been done in the description of the molecular spectrum of molecules. But it is still the only model that describes almost all levels of baryons in a consistent way and is able to predict the energies of levels yet to be found experimentally.

Acknowledgements - I thank the comments of the referee Prof. José Muñoz.

[1] S Gasiorowicz, J L Rosner, Hadron spectra and quarks, Am. J. Phys. 49, 954 (1981).

[2] N Isgur, G Karl, P-wave baryons in the quark model, Phys. Rev. D 18, 4187 (1978).
[3] S Capstick, N Isgur, Baryons in a relativized quark model with chromodynamic, Phys. Rev. D 34, 2809 (1986).

[4] R K Bhaduri, B K Jennings, J C Waddington, Rotational bands in the baryon spectrum, Phys. Rev. D 29, 2051 (1984).

[5] M V N Murthy, M Dey, R K Bhaduri, Rotational bands in the baryon spectrum. II, Phys. Rev. D 30, 152 (1984).

[6] M V N Murthy, M Brack, R K Bhaduri, B K Jennings, The spin-orbit puzzle in the spectra and deformed baryon model, Z. Phys. C 29, 385 (1985).

[7] P Stassart, F Stancu, J-M Richard, L Theu $\beta 1$, On the scalar meson exchange in the baryon spectra, J. Phys. G: Nucl. Partic. 26, 397 (2000).

[8] A Hosaka, H Toki, M Takayama, Baryon spectra in deformed oscillator quark model, Mod. Phys. Lett. 13, 1699 (1998).

[9] M E de Souza, Calculation of the energy levels and sizes of baryons with a noncentral harmonic potential, arXiv:hep-ph/0209064v1 (2002).

[10] M E de Souza, The energies of baryons, In: Proceedings of the XIV Brazilian National Meeting of the Physics of Particles and Fields, Eds. A J da Silva, A Suzuki, C Dobrigkeit, C Z de Vasconcelos, C Wotzacek, R F Ribeiro, S $\mathrm{R}$ de Oliveira, S A Dias, Pag. 331, Sociedade Brasileira de Física, São Paulo (1993).

[11] K Nakamura et al.(Particle Data Group), Review of particle physics, J. Phys. G: Nucl. Partic. 37, 075021 (2010).

[12] R Shankar, Principles of quantum mechanics, Plenum Press, New York (1994).

[13] W Greiner, J A Maruhn, Nuclear models, Springer, Berlin (1996).

[14] G Herzberg, Infrared and Raman spectra, Van Nostrand Heinhold Company, New York (1945).

[15] L. Bai-Qing and C. Kuang-Ta, Bottomonium spectrum with screeened potential, Commun. Theor. Phys. 52, 653 (2009). 
Papers in Physics, vol. 3, ART. 030003 (2011) / M. E. de Souza

[16] H Hassanabadi, A A Rajabi, Determination of the potential coefficients of the baryons and the effect of spin and isospin potential on their energy, In: 11th Internation Conference on
meson-Nucleon Physics and Structure of the Nucleon, Eds. H Machner, S Krewald, Pag. 128, IKP, Forschungzentrum Jülich (2007). 